\title{
OTIMIZAÇÃO DE METODOLOGIA PARA DERIVAÇÃO DE DESOXINIVALENOL ATRAVÉS DE PLANEJAMENTO EXPERIMENTAL
}

\author{
Jaqueline Garda* e Eliana Badiale-Furlong \\ Departamento de Química, Fundação Universidade Federal do Rio Grande, CP 474, 96201-900 Rio Grande - RS, Brasil
}

Recebido em 8/1/07; aceito em 13/7/07; publicado na web em 19/12/07

\begin{abstract}
OPTIMIZATION OF THE METHODOLOGY FOR DEOXYNIVALENOL DERIVATIZATION BY EXPERIMENTAL PLANNING. The objective of this work was to optimize the derivatization reaction for determining deoxynivalenol (DON) by gas chromatography employing an experimental planning procedure. The factors were: temperature, reaction time, catalyst and trifluoroacetic anhydride concentration. The relative peak areas were used to evaluated the effects. The best conditions for DON derivatization were $200 \mu \mathrm{L}$ TFAA and $18 \mathrm{mg}$ sodium bicarbonate for $6 \mathrm{~min}$ at $74{ }^{\circ} \mathrm{C}$ for 7 to $21 \mu \mathrm{g}$ of DON. Under these conditions, the detection limit was $1.4 \mu \mathrm{g}$ of DON.
\end{abstract}

Keywords: deoxynivalenol; derivatization; methodology.

\section{INTRODUÇÃO}

A molécula de desoxinivalenol (DON) possui uma estrutura de um sesquiterpenóide tetracíclico com sete centros estéreos. Sua fórmula empírica é $\mathrm{C}_{15} \mathrm{H}_{20} \mathrm{O}_{6}$, nomeado como 12,13-epoxi-3,7,15triidroxi-tricotec-9-en-8-ona, $(3 \alpha, 7 \alpha)-(9 \mathrm{CI})$. Esta estrutura é produzida por diversas espécies fúngicas do gênero Fusarium, um contaminante natural de cereais e seus resíduos, e até em vegetais intactos $^{1-3}$.

Além do gênero Fusarium, outros como Mirothecium, Cephalosporium, Verticimosporium e Stachybotrys podem ser produtores desta micotoxina, podendo ocorrer em pré ou pós-colheita, em temperatura entre 0 a $35{ }^{\circ} \mathrm{C}$ e umidade relativa entre 80 e $90 \% \%^{3,4}$.

Este composto se caracteriza pela estabilidade, que pode ser verificada durante muitos anos quando estocado a temperatura ambiente, mesmo aquecido a $135{ }^{\circ} \mathrm{C}$ não sofre degradação e, portanto, também em condições ambientais usuais de processamento e preparo de alimentos ${ }^{5,6}$. O DON, como outros tricotecenos, não é volátil e pode ser desativado sob condições drásticas ácidas ou alcalinas, em presença de hidretos de alumínio, lítio ou peróxidos e hidratação em autoclave, onde o anel epóxido pode sofrer abertura. A enzima epóxido hidrase também é capaz de abrir o anel e produzir glicóis ${ }^{6,7}$. Fungos e bactérias podem também alterar a estrutura deste tricoteceno, detoxificando- ${ }^{8}$.

Desde a demonstração da toxicidade dos tricotecenos, o desenvolvimento de técnicas aplicadas na detecção, análise e caracterização cromatográfica vem sendo grandemente incentivada pela possibilidade de separar diferentes metabólitos simultaneamente em suas matrizes de origem. Várias metodologias para determinação de tricotecenos tem sido revisada por autores, tais como Betina ${ }^{9}$, Scott ${ }^{10,11}$, Langseth e Rungberget ${ }^{12}$, Placinta D`Mello e Macdonald ${ }^{13}$, Kotal et al. ${ }^{14}$, Koch $^{15}$, Murphy et al. ${ }^{16}$.

A avaliação destes informes demonstra que as etapas analíticas para determinação de micotoxinas consistem nas etapas de amostragem, extração, limpeza, concentração, separação dos componentes do extrato, detecção, confirmação de identidade e quantificação. Não diferindo, portanto, das marchas analíticas para outros tipos de contaminantes incidentais. A detecção desta toxina é dificultada pela baixa absortividade no ultravioleta e fluorescência,

*e-mail: jaquelinegarda@yahoo.com.br o que torna a cromatografia gasosa uma das técnicas preferenciais para a sua determinação ${ }^{16,17}$.

A derivação, ponto crítico na determinação de DON, antecede ao processo cromatográfico para reduzir a polaridade dos grupamentos livres, aumentar a pressão de vapor dos compostos, possibilitar a separação de substâncias muito semelhantes entre si no estado natural e introduzir grupos que tornam o composto adequado ao sistema de detecção empregado ${ }^{14,17,18}$.

A derivação do grupo hidroxila do DON é fundamental, pois a sua volatilidade pode ser aumentada substituindo o hidrogênio de grupos funcionais, por radicais do tipo dimetilsilil, trimetilsilil, butirilsilil e flúor ${ }^{19}$. Estão descritos diferentes sistemas para derivação empregando anidrido trifluoroacético, heptafluoro-butirilimidazol, pentafluorobutilimidazol, trimetilsilil, bis-trimetilsililacetamida, hexametildisilazano e trimetilsilimidazol, sendo que melhores resultados foram conseguidos com heptafluoro-butiril e anidrido trifluoroacético, utilizando como catalisador a piridina ou bicarbonato $^{10,17,20,21}$.

No entanto, a maioria dos derivatizantes não estão disponíveis no comércio nacional, o que dificulta o estabelecimento de rotinas analíticas acessíveis para a maioria dos laboratórios envolvidos com a detecção desta micotoxina. Além disso, halogenados dificultam a detecção em detectores de ionização de chama e os sililizantes são altamente corrosivos. O emprego destes reagentes em termos de quantidades, relação com catalisadores e tempo/temperatura de reação são pouco estudados. Faltam, portanto, dados sobre os efeitos destas variáveis nos resultados quali e quantitativos da micotoxina que torne o emprego destes reagentes comparáveis aos recomendados por órgãos oficiais. Estas dificuldades podem ser visualizadas na avaliação das poucas informações disponíveis no país sobre a ocorrência de DON em alimentos destinados ao consumo humano, a não existência de barreiras legais a comercialização de produtos contaminados ou medidas preventivas a contaminação.

As informações disponíveis na literatura permitem estimar que $25 \%$ dos produtos agrícolas cultivados mundialmente estejam contaminados com desoxinivalenol, o que torna imprescindível dispor de dados específicos e confiáveis para estimar o risco ao consumo de alimentos ou insumos contaminados por esta toxina ingeridos diariamente. A partir deste conhecimento, podem ser adotadas medidas preventivas eficientes para minimizar danos à saúde de humanos e animais de criação ${ }^{1}$. 
Este trabalho pretende contribuir com a disponibilização de metodologia otimizada de derivação para a determinação de desoxinivalenol em fase gasosa para instrumentos acoplados com detector do tipo universal.

\section{PARTE EXPERIMENTAL}

\section{Reagentes}

O padrão de DON e padrão interno, metil éster de ácido aracdônico ( $90 \%$ de pureza) utilizado a uma concentração de 0,1 $\mathrm{mg} / \mathrm{mL}$ foram adquiridos da Sigma Chemical Company (EUA). O anidrido trifluoroacético (TFAA) com 99\% de pureza e densidade de $1,51 \mathrm{~g} / \mathrm{mL}$ foi da marca Merck (Alemanha).

Outros reagentes e solventes utilizados durante os testes foram de grau p.a.

\section{Padrão de desoxinivalenol}

Para o preparo da solução estoque de DON, a toxina foi dissolvida em benzeno: acetonitrila (95:5) resultando em uma concentração de $100 \mu \mathrm{g} / \mathrm{mL}$, conforme Shepeherd e Gilbert ${ }^{22}$. A solução de trabalho consistiu na diluição da solução estoque para concentração de $50 \mu \mathrm{g} / \mathrm{mL}$. A determinação da concentração foi estimada pela relação p/v utilizado na preparação da solução e confirmada pelo procedimento descrito por Bennett e Shotwell ${ }^{23}$ utilizando a absortividade molar do padrão 219 para DON em espectrofotômetro modelo Cary 100 da Varian (EUA).

\section{Padronização de metodologia para derivação}

A partir de condições já estudadas por Garda, Macedo e BadialeFurlong ${ }^{24}$, Milanez, Valente-Soares e Baptista ${ }^{18}$ foram estudadas as variáveis: tempo de reação (30, 45 e $60 \mathrm{~min})$, temperatura (40, 60 e $80{ }^{\circ} \mathrm{C}$ ) e catalisador (bicarbonato de sódio e piridina), através de planejamento fatorial $2^{3}$, ou seja, três variáveis ou fatores testados em dois níveis $(-1$ e +1$)$ e 6 pontos centrais (0), gerando 14 experimentos (Tabela 1) ${ }^{25,26}$. Para estabelecer a melhor condição de tempo e temperatura, utilizou-se planejamento fatorial completo $2^{2}$, duas variáveis em dois níveis $(-1$ e +1$)$, com pontos axiais $(-1,414$ $\mathrm{e}+1,414)$ e 3 centrais (0) (Tabela 2).

Tabela 1. Valores de área relativa para o planejamento fatorial $2^{3}$ e 6 pontos centrais (a)

\begin{tabular}{lcccr}
\hline \multicolumn{2}{l}{ Experimento } & \multicolumn{3}{c}{ Variáveis } \\
& $\begin{array}{c}\text { Tempo } \\
(\mathrm{min})\end{array}$ & $\begin{array}{c}\text { Temperatura } \\
\left({ }^{\circ} \mathrm{C}\right)\end{array}$ & Catalisador & $\begin{array}{r}\text { Área DON/ } \\
\text { área ácido } \\
\text { aracdônico }\end{array}$ \\
\hline 1 & $-1(30)$ & $-1(40)$ & 1 (Piridina) & 0,00 \\
2 & $1(60)$ & $-1(40)$ & $-1\left(\mathrm{NaHCO}_{3)}\right.$ & 0,38 \\
3 & $-1(30)$ & $1(80)$ & $-1\left(\mathrm{NaHCO}_{3}\right)$ & 0,34 \\
4 & $1(60)$ & $1(80)$ & 1 (Piridina) & 0,04 \\
5 & $-1(30)$ & $-1(40)$ & $-1\left(\mathrm{NaHCO}_{3)}\right.$ & 0,03 \\
6 & $1(60)$ & $1(80)$ & -1 (NaHCO & 0,26 \\
7 & $-1(30)$ & $1(80)$ & 1 (Piridina) & 0,24 \\
8 & $1(60)$ & $-1(40)$ & 1 (Piridina) & 0,00 \\
9 & $0(45)$ & $0(60)$ & 0 (NaHCO & 0,00 \\
10 & $0(45)$ & $0(60)$ & 0 (NaHCO & 0,00 \\
11 & $0(45)$ & $0(60)$ & 0 (NaHCO & 0,00 \\
12 & $0(45)$ & $0(60)$ & 0 (Piridina) & 0,15 \\
13 & $0(45)$ & $0(60)$ & 0 (Piridina) & 0,16 \\
14 & $0(45)$ & $0(60)$ & 0 (Piridina) & 0,12 \\
\hline
\end{tabular}

Tabela 2. Valores de área relativa para o planejamento fatorial $2^{2}$ com pontos axiais e 3 pontos centrais (b)

\begin{tabular}{|c|c|c|c|}
\hline \multicolumn{2}{|c|}{ Experimento } & \multicolumn{2}{|l|}{ Variáveis } \\
\hline & $\begin{array}{c}\text { Temperatura } \\
\left({ }^{\circ} \mathrm{C}\right)\end{array}$ & $\begin{array}{l}\text { Tempo } \\
\text { (min) }\end{array}$ & $\begin{array}{l}\text { Área DON/área } \\
\text { ácido aracdônico }\end{array}$ \\
\hline 1 & $-1(80)$ & $-1(10)$ & 0,206 \\
\hline 2 & $1(105)$ & $-1(10)$ & 0,081 \\
\hline 3 & $-1(80)$ & $1(30)$ & 0,296 \\
\hline 4 & $1(105)$ & $1(30)$ & 0,073 \\
\hline 5 & $-1,414(74)$ & $0(20)$ & 0,065 \\
\hline 6 & 1,414 (116) & $0(20)$ & 0,040 \\
\hline 7 & $0(95)$ & $-1,414(6)$ & 0,314 \\
\hline 8 & $0(95)$ & $1,414(34)$ & 0,048 \\
\hline 9 & $0(95)$ & $0(20)$ & 0,145 \\
\hline 10 & $0(95)$ & $0(20)$ & 0,158 \\
\hline 11 & $0(95)$ & $0(20)$ & 0,200 \\
\hline
\end{tabular}

Obtido o melhor catalisador, os valores ótimos para tempo de reação e temperatura, foi estudado a quantidade de TFAA, agente derivatizante, e quantidade de catalisador bicarbonato de sódio através do planejamento completo $2^{2}$ com pontos axiais e dois centrais (Tabela 3).

Tabela 3. Planejamento fatorial $2^{2}$ com pontos axiais e 2 centrais (c)

\begin{tabular}{|c|c|c|c|}
\hline \multicolumn{2}{|c|}{ Experimento } & \multicolumn{2}{|l|}{ Variáveis } \\
\hline & $\begin{array}{l}\text { TFAA } \\
(\mu \mathrm{L})\end{array}$ & $\begin{array}{l}\mathrm{NaHCO}_{3} \\
(\mathrm{mg})\end{array}$ & $\begin{array}{l}\text { Área DON/área } \\
\text { ácido aracdônico }\end{array}$ \\
\hline 1 & $-1(100)$ & $-1(30)$ & 0,022 \\
\hline 2 & $1(300)$ & $-1(30)$ & 0,000 \\
\hline 3 & $-1(100)$ & $1(90)$ & 0,000 \\
\hline 4 & $1(300)$ & $1(90)$ & 0,031 \\
\hline 5 & $-1,414(59)$ & $0(60)$ & 0,000 \\
\hline 6 & 1,414 & $0(60)$ & 0,103 \\
\hline 7 & $0(200)$ & $-1,414(18)$ & 0,166 \\
\hline 8 & $0(200)$ & $1,414(102)$ & 0,018 \\
\hline 9 & $0(200)$ & $0(60)$ & 0,043 \\
\hline 10 & $0(200)$ & $0(60)$ & 0,056 \\
\hline
\end{tabular}

Para análise estatística dos dados foi utilizado o programa Statistica 6.0, considerando a área relativa da toxina (área DON/ área ácido aracdônico) como resposta das variáveis em estudo.

\section{Quantificação por cromatografia gasosa}

Após a realização das derivações sob as diferentes condições em questão, o derivatizado foi seco sob nitrogênio, seguido de adição de $700 \mu \mathrm{L}$ de tolueno e $2 \mathrm{~mL}$ de água destilada, agitando-se a mistura em banho ultrasônico. O sistema foi congelado para melhor separação das fases, sendo retirados $500 \mu \mathrm{L}$ da fração de solvente, que foi seca sob nitrogênio. O resíduo foi ressuspenso em hexano e adicionado padrão interno metil éster ácido aracdônico $(1 \mu \mathrm{g})$, sendo injetado $1 \mu \mathrm{L}$ em cromatógrafo gasoso.

A quantificação foi realizada em cromatógrafo gasoso Varian 3400 equipado com injetor "split/splitless" e detector de ionização de chama, coluna DB-17 (J\&W Scientific - EUA) de 30 m, com $0,25 \mathrm{~mm}$ de d.i. e $0,25 \mu \mathrm{m}$ de filme de 50\%-fenil metilpolisiloxano. O equipamento foi monitorado pelo software Star Chromatography Workstation, versão 4.1, marca Varian. 
Tabela 4. Efeitos e coeficientes para variáveis significativas obtidas pelo tratamento estatístico

\begin{tabular}{llllll}
\hline Planejamento & Variável & Efeito & $\begin{array}{l}\text { Desvio } \\
\text { padrão }\end{array}$ & $\begin{array}{l}\text { Nível de } \\
\text { significância (p) }\end{array}$ & Coeficientes \\
\hline (a) & Média/interação & 0,12868 & 0,021198 & 0,002154 & 0,122868 \\
& catalisador & $-0,179964$ & 0,056085 & 0,023762 & $-0,089982$ \\
& 1 x 2 & $-0,155949$ & 0,056085 & 0,038876 & 0,077975 \\
\hline (b) & Média/interação & 0,1850 & 0,0122 & 0,0044 & 0,1850 \\
& temperatura (Q) & $-0,1028$ & 0,0235 & 0,0483 & $-0,0514$ \\
& Tempo (L) & $-0,1185$ & 0,0206 & 0,0290 & $-0,0592$ \\
& 1 x 2 & 0,1739 & 0,0292 & 0,0270 & 0,0869 \\
\hline (c) & Média/interação & 0,0439 & 0,0029 & 0,0421 & 0,0439 \\
& TFAA (L) & 0,0387 & 0,0065 & 0,1060 & 0,0193 \\
& NaHCO (L) & $-0,0501$ & 0,0065 & 0,0822 & $-0,0250$ \\
& TFAA x NaHCO & 0,0265 & 0,0092 & 0,2125 & 0,0132 \\
\hline
\end{tabular}

*Não utilizado para cálculos de ANOVA. (a) Para tempo, temperatura e catalisador utilizando planejamento fatorial $2^{3}$ e 6 pontos centrais; (b) para temperatura e tempo utilizando planejamento fatorial $2^{2}$ com pontos axiais e 3 pontos centrais; (c) para quantidade de TFAA e $\mathrm{NaHCO}_{3}$ utilizando planejamento fatorial $2^{2}$ com pontos axiais e 2 pontos centrais.

As condições cromatográficas empregadas foram: temperatura do injetor $250{ }^{\circ} \mathrm{C}$, abertura da válvula a 0,75 min, fluxo de limpeza do injetor de $75 \mathrm{~mL} /$ minuto, detector $300{ }^{\circ} \mathrm{C}$, atenuação de $16 \mathrm{x}$ $10^{12}$. A programação para a coluna cromatográfica foi de $100{ }^{\circ} \mathrm{C}$ durante 1 min aumentando $50{ }^{\circ} \mathrm{C} / \mathrm{min}$ até $200{ }^{\circ} \mathrm{C}$, permanecendo 2 min, em seguida aumentando $4{ }^{\circ} \mathrm{C} / \mathrm{min}$ até $250{ }^{\circ} \mathrm{C}$, permanecendo 11,5 min, completando 29 min de corrida cromatográfica.

\section{Confirmação do tempo de retenção, limite de quantificação, detecção e linearidade}

Para a confirmação da presença da toxina no sistema foi realizada uma reação de redução de DON com $200 \mu \mathrm{L}$ de solução de boridreto de sódio $(2 \mathrm{mg} / \mathrm{mL})$, deixando reagir à temperatura ambiente por 24 h. Esta solução foi neutralizada com ácido clorídrico 2 $\mathrm{M}$, seguida da evaporação ${ }^{16}$ e derivação com TFAA em meio alcalino.

Os limites de detecção e linearidade da concentração x área relativa de pico foram determinadas pela injeção de soluções padrões. O limite de detecção foi estabelecido pela injeção de concentrações de padrões que gerassem um sinal no detector três vezes superior ao desvio padrão das áreas relativas no tempo de retenção da toxina quando se injetava um branco de derivação $0^{27,28}$.

A linearidade foi determinada a partir da injeção de soluções padrões de DON com concentrações variando entre 1,4 a $21 \mu \mathrm{g}$, com emprego de padrão interno estimando a área relativa como resposta a variação de concentração de padrão.

\section{RESULTADOS E DISCUSSÃO}

Através das condições cromatográficas citadas, o tempo de retenção de desoxinivalenol foi de 7,4 min confirmado por comparação com a alteração do tempo de retenção do padrão de DON reduzido.

O planejamento experimental foi utilizado para possibilitar a inferência estatística e fornecer informações sobre o comportamento das respostas entre os níveis das variáveis em estudo, evidenciando a qualidade da repetibilidade, minimizando custos ao não se repetir todos experimentos, demanda e desgaste de equipamento e tempo, menor exposição do analista e menor volume de descarte. Esta ferramenta melhorou a qualidade da informação através dos resultados, permitindo otimizar e construir o modelo preditivo dentro da faixa ótima para a reação de derivação, através da avaliação do erro experimental estimando a reprodutibilidade dos resultados alcançados ${ }^{25,26}$.
A primeira etapa de estudo do efeito das variáveis tempo, temperatura e eficiência do catalisador, tem seus resultados apresentados na Tabela 1 como área relativa DON/ácido aracdônico. Este foi escolhido como padrão interno por sua estabilidade frente a condições cromatográficas, tempo de retenção e disponibilidade no mercado nacional e custo acessível.

A análise estatística mostrou que ocorreu um efeito significativo antagônico (Tabela 4) para catalisador $(\mathrm{p}=0,024)$ e para a interação entre tempo e temperatura $(\mathrm{p}=0,039)$.

Quando o catalisador piridina foi utilizado, a área relativa do pico detectado foi menor comparada à área relativa obtida com bicarbonato de sódio $(-0,18)$. Este resultado concorda com outros descritos na literatura como Nunes et al. ${ }^{29}$, Oliveira et al. ${ }^{28}$, Milanez et al. ${ }^{18}$. No entanto, Gilbert et al. ${ }^{20}$, Scott ${ }^{10}$, Kientz e Verweij ${ }^{30}$ encontraram resultados contrários utilizando detector de captura de elétrons e/ou de massas.

Considerando a interação tempo e temperatura variáveis significativas para a eficiência da reação, foi realizado um planejamento completo com pontos axiais e centrais para obtenção do ponto ótimo de reação (Tabelas 2 e 4). Este permitiu otimizar e construir modelo preditivo dentro da faixa ótima para a reação de derivação e conforme o previsto, efeito significativo ocorreu para as duas variáveis e a interação.

As variáveis estudadas isoladamente mostraram que quanto maior o intervalo de tempo e temperatura testados, menores foram às áreas relativas do pico detectada. Isto pode indicar que estão sendo formados outros compostos decorrentes da fragmentação do derivatizado tanto pelo longo período de exposição ao meio quanto pela alta temperatura. A interação destas duas variáveis apresentou o efeito significativo previsto, demonstrando que em menores tempos e temperaturas de reação, maiores foram as áreas detectadas. Estes resultados foram significativos e preditivos para a ação combinada destes parâmetros nas áreas relativas detectadas (Tabela 5), originando a Equação 1 e Figura 1.

Área relativa detectada $=0,1850-0,0514 \mathrm{x}$ temperatura ${ }_{\text {COD }}-0,0592 \mathrm{x}^{\text {tempo }}$ COD $+0,0869 \mathrm{x}$ temperatura $\mathrm{x}$ tempo ${ }_{\mathrm{COD}}$

Nas condições ótimas para tempo e temperatura, o estudo da quantidade de agente derivatizante, anidrido trifluoroacético (TFAA) a ser adicionado, e a quantidade de catalisador, bicarbonato de sódio $\left(\mathrm{NaHCO}_{3}\right)$ como determinado anteriormente, permitiu 
Tabela 5. Dados da ANOVA para planejamento experimental avaliando tempo, temperatura e catalisador

\begin{tabular}{|c|c|c|c|c|c|c|}
\hline Planejamento & $\begin{array}{l}\text { Fonte de } \\
\text { variação }\end{array}$ & $\begin{array}{c}\text { Soma } \\
\text { quadrática }\end{array}$ & $\begin{array}{l}\text { Graus de } \\
\text { liberdade }\end{array}$ & $\begin{array}{l}\text { Média } \\
\text { quadrática }\end{array}$ & Teste F & $\mathrm{F}_{\text {tab }(95 \%)}$ \\
\hline \multirow[t]{5}{*}{ (a) } & Regressão & 0,113414 & 2 & 0,056707 & 5,109 & 3,98 \\
\hline & Resíduo & 0,122085 & 11 & 0,0111 & & \\
\hline & Falta de ajuste & 0,090629 & 6 & & & \\
\hline & Erro puro & 0,031456 & 5 & & & \\
\hline & Total & 0,235499 & 13 & & & \\
\hline \multirow[t]{5}{*}{ (b) } & Regressão & 0,0746 & 3 & 0,0249 & 8,89 & 4,35 \\
\hline & Resíduo & 0,0197 & 7 & 0,0028 & & \\
\hline & Falta de ajuste & 0,0180 & 5 & & & \\
\hline & Erro puro & 0,0017 & 2 & & & \\
\hline & Total & 0,0943 & 10 & & & \\
\hline \multirow[t]{5}{*}{ (c) } & Regressão & 0,0079 & 2 & 0,00395 & 0,223 & $49,50_{(90 \%)}$ \\
\hline & Resíduo & 0,0177 & 1 & 0,0177 & & \\
\hline & Falta de ajuste & 0,0176 & 6 & & & \\
\hline & Erro puro & 0,0001 & 1 & & & \\
\hline & Total & 0,0256 & 9 & & & \\
\hline
\end{tabular}

(a) Para tempo, temperatura e catalisador utilizando planejamento fatorial $2^{3}$ e 6 pontos centrais; (b) para temperatura e tempo utilizando planejamento fatorial $2^{2}$ com pontos axiais e 3 pontos centrais; (c) para quantidade de TFAA e $\mathrm{NaHCO}_{3}$ utilizando planejamento fatorial $2^{2}$ com pontos axiais e 2 pontos centrais.

verificar que para o derivatizante, o intervalo significativo foi de 100 a $300 \mu \mathrm{L}(\mathrm{p}=0,11)$, e o aumento da adição deste agente foi acompanhado por uma maior $(0,0387)$ área relativa detectada.

Para bicarbonato de sódio, a adição de maior quantidade na proporção de 30 para $90 \mathrm{mg}$ causou um efeito significativo $(\mathrm{p}=0,0029)$. Este aumento resultou em uma diminuição da área relativa do pico. Isto foi verificado por $\mathrm{Scott}^{10}$ e Garda et al ${ }^{24}$, demonstrando que a utilização em excesso de bicarbonato como meio de reação poderia causar diminuição no seu rendimento possivelmente pela reversão da reação de derivação.

Estes resultados significativos correspondem a equação preditiva (Equação 2) para os parâmetros de agente derivatizante TFAA e catalisador bicarbonato de sódio quanto a área relativa.

Área relativa detectada $=0,0439+$

$0,0193 \times$ TFAA $_{\mathrm{COD}}-0,0250 \times \mathrm{NaHCO}_{3 \mathrm{COD}}$

Estes dados confirmam a utilização de $30 \mathrm{mg}$ de bicarbonato de sódio e $300 \mu \mathrm{L}$ de TFAA anteriormente utilizados por Nunes $e t$ al. ${ }^{29}$, Garda et al. ${ }^{24}$, Milanez et al..$^{31}$, Oliveira et al. ${ }^{28}$.

O emprego do intervalo ótimo para a reação de derivação, no entanto, utiliza a interação entre os componentes da reação, que para agente derivatizante e catalisador $(\mathrm{p}=0,2125)$ ocasiona um aumento na área relativa quando comparada com variáveis isoladas, indicando que $18 \mathrm{mg}$ de bicarbonato de sódio e $200 \mu \mathrm{L}$ de TFAA seriam as melhores condições reacionais similar as utilizados por Milanez et al. ${ }^{31}$. O que é bastante interessante, pois menor é o gasto de material, menor a geração de resíduos para descarte e menores são os sinais espúrios no cromatograma (Figura 3 ).

\section{Confirmação do tempo de retenção, limite de quantificação,} detecção e linearidade

A reação de redução para confirmar a presença de DON mostrou que o padrão reduzido apresentava respostas semelhantes às condições de derivação otimizadas, apresentando apenas um aumento no tempo de retenção relativo.

A linearidade da curva padrão foi realizada nas condições ótimas de derivação: adição de $18 \mathrm{mg}$ de bicarbonato de sódio, $200 \mu \mathrm{L}$

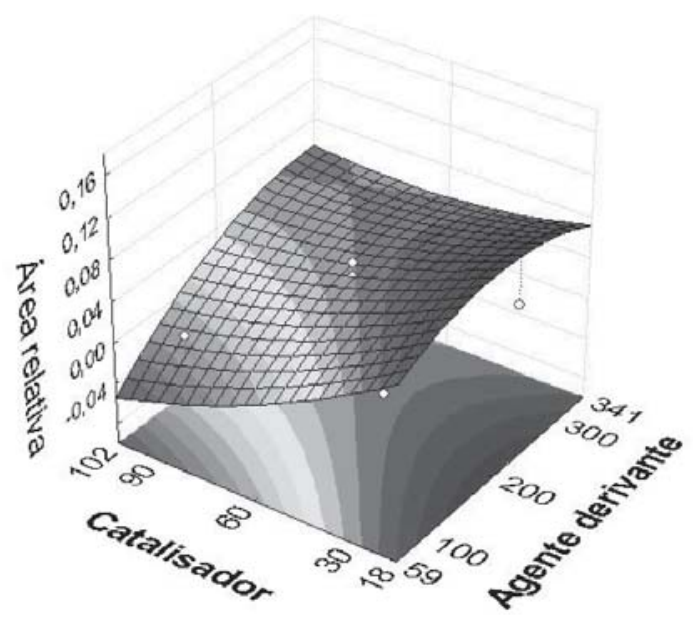

Figura 1. Superfície de resposta para temperatura e tempo

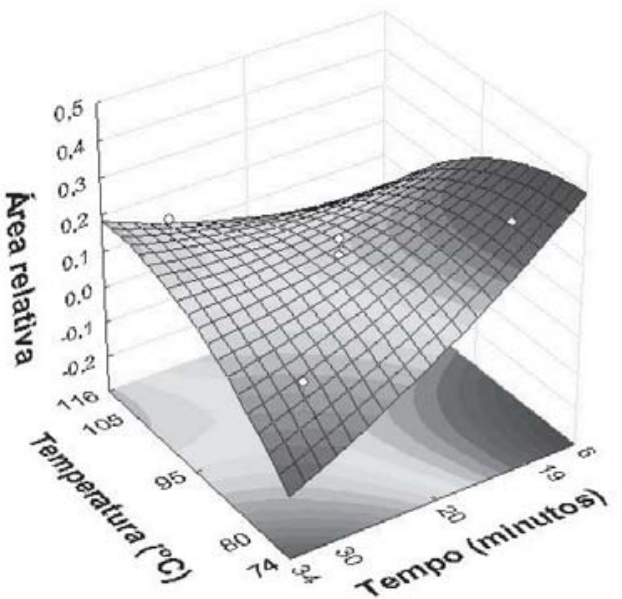

Figura 2. Superfície de resposta para agente derivatizante e catalisador 


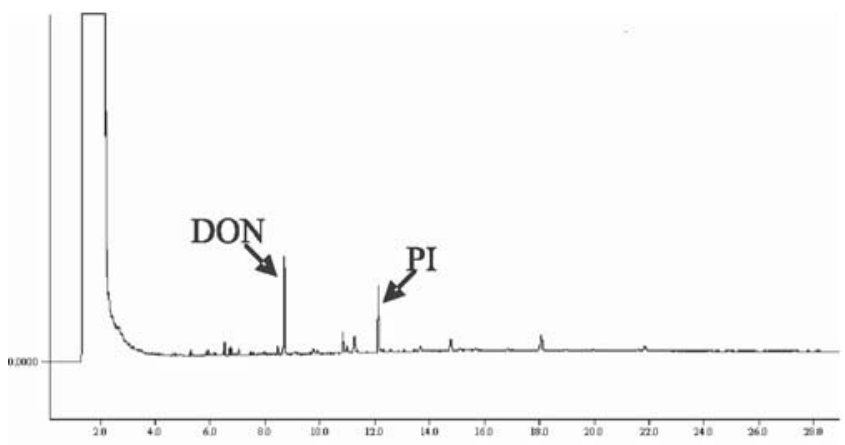

Figura 3. Cromatograma de desoxinivalenol com adição de padrão interno.

de TFAA, reação a $74{ }^{\circ} \mathrm{C}$ durante 6 min. A linearidade da curva foi significativa a partir de $50 \mathrm{ng} / \mu \mathrm{L}$ ou $7 \mu \mathrm{g}$ de desoxinivalenol, correspondendo os pontos de 7,10,13,15,18, $21 \mu \mathrm{g}$, preparados em triplicatas em ordem randômica tendo um $\mathrm{R}^{2}=0,9852^{32}$.

$\mathrm{O}$ limite de detecção determinado pelas sucessivas diluições foi de $10 \mathrm{ng} / \mu \mathrm{L}$ gerando um sinal do detector três vezes superior ao desvio padrão das áreas relativas no tempo de retenção das toxinas quando se injetou branco de derivação. Esta concentração correspondeu a 1,4 $\mu \mathrm{g}$ de desoxinivalenol derivado, valor que permite detectar DON contaminando amostras em concentrações 10 vezes inferiores aos estabelecidos pelas legislações de vários países.

\section{CONCLUSÃO}

Empregando três etapas de planejamento experimental para a reação de derivação pela acetilação, foi possível determinar as condições ótimas de reação para emprego das variáveis tempo, temperatura, agente derivatizante e catalisador com resultados significativos e preditivos para área relativa detectada. As condições otimizadas ocorriam quando foram derivados de 1,4 a $21 \mu \mathrm{g}$ de DON com adição de $18 \mathrm{mg}$ de bicarbonato de sódio e $200 \mu \mathrm{L}$ de TFAA, reagindo durante 6 min a $74{ }^{\circ} \mathrm{C}$.

\section{REFERÊNCIAS}

1. Meky, F. A.; Hardie, L. J.; Evans, S.W.; Wild, C. P.; Food Chem. Toxicol. 2001, 39, 827.
2. Atroshi, F.; Rizzo, A.; Weastermarck, T.; Ali-Vehmas, T.; Toxicology, 2002, $180,151$.

3. Sudakin, D. L.; Toxicol. Lett. 2003, 143, 97.

4. Sweeney, M. J.; Dobson, A. D. W.; Int. J. Food Microbiology 1998, 43, 141.

5. Yoshisawa, T.; Mycotoxin Analysis for Federative republic of Brazil, Training Course, Japão, 2001, p. 283.

6. Moss, M. O.; Thrane, U.; Toxicol. Lett. 2004, 153, 23.

7. Pronik, C.; Cenkowski, S.; Abransom, D.; Food Control 2006, 17, 789.

8. Ueno, Y. Em Developments in Food Science; Ueno, Y. ed.; Elsevier: Tokyo, 1983, vol. 4.

9. Betina, V.; J. Chromatogr. 1985, 334, 211.

10. Scott, P. M. Em Chromatography of Mycotoxins: techniques and Applications; Betina, V., ed.;Elsivier: Amsterdam, 1991.

11. Scott, P. M.; Kanhere, S. R.; Daley, E. F.; Faber, J. M.; Food Addit. Contam., 1993, 10, 381.

12. Langset, W.; Rundberget, T. ; J. Chromatogr., A 1998, 815, 103.

13. Placinta, C. M.; D’Mello, J. P. F.; Macdonald, A. M. C.; Animal Feed Science and Technology 1999, 78, 21.

14. Kotal, F.; Holadová, K.; Hajslová, J.; Poustka, J.; Radová, Z.; J. Chromatogr., A 1999, 830, 219.

15. Koch, P.; Toxico. Lett. 2004, 153, 109.

16. Murphy, P. A.; Hendrich, S.; Landgren, C.; Bryant, C.; J. Food Sci. 2006, $71,51$.

17. Badiale-Furlong, E.; Soares, L. M. V.; J. AOAC Int. 1995, 78, 386.

18. Milanez, T. V.; Valente-Soares, L. M.; Baptista, G. G.; Rev. Inst. Adolfo Lutz 2004, 63, 15.

19. Scott, P. M.; J. AOAC Int. 1982, 65, 876.

20. Gilbert, J.; Startin, J. R.; Crews, C.; J. Chromatogr. 1984, 319, 376.

21. Garda, J.; Macedo, R. M.; Faria, R.; Bernd, L.; Dors, G. C.; BadialeFurlong, E.; Food Control 2005, 16, 423.

22. Shepherd, M. J.; Gilbert, J.; J. Agric. Food Chem. 1988, 36, 305.

23. Bennet, G. A.; Shotwell, O. L.; J. AOAC Int. 1990, 73, 270.

24. Garda, J.; Macedo, R. M.; Badiale-Furlong, E.; Ciên. Tecnol. Aliment. 2004, $24,657$.

25. Neto, B. B.; Scarminio, I. S.; Bruns, R. E.; Como fazer experimentos: pesquisa e desenvolvimento na ciência e na indústria, $2^{\mathrm{a}}$ ed., Editora da UNICAMP: Campinas 2003.

26. Rodrigues, M. I.; Iemma, A. F.; Planejamento de experimentos e otimização de processos:uma estratégia seqüencial de planejamentos, $1^{\mathrm{a}}$ ed., SP: Casa do Pão Editora: Campinas 2005.

27. Caulcutt, R.; Boddy, R.; Statistic for analytical Chemists, Chapman and Hall: London, 1983.

28. Oliveira, A. Q.; Soares, L. M. V.; Sawazaki, E.; Ciênc. Tecnol. Aliment. 2001, 21, 330.

29. Nunes, I. L.; Maganin, G.; Bertolin, T. E.; Badiale-Furlong, E.; Ciên. Tecnol. Aliment. 2003, 23, 190.

30. Kientz, C. E.; Verweij, A.; J. Chromatogr. 1986, 355, 229.

31. Milanez, T. V.; Valente-Soares, L. M.; Baptista, G. G.; Food Control 2006, $17,293$.

32. Thompson, M.; Ellison, S. L. R.; Wood, R.; Pure Appl. Chem. 2002, 74, 835. 